\title{
Hue and Saturation in the RGB Color Space
}

\author{
Martin Loesdau, Sébastien Chabrier, and Alban Gabillon \\ Laboratoire d'Excellence CORAIL \\ Géopôle du Pacifique Sud EA4238 \\ Université de la Polynésie française, Tahiti \\ \{martin.loesdau, sebastien.chabrier, alban.gabillon\}@upf.pf
}

\begin{abstract}
While the RGB color model refers to the biological processing of colors in the human visual system, the HSV color model corresponds to the human perception of color similarity. In this paper we formulate a projection of RGB vectors within the RGB color space, which separates achromatic from chromatic information. The projection is the mathematical equivalent to Hue and Saturation of the HSV color space in the RGB space. It integrates the psycho-visual concept of human differentiation between colors of the HSV space into the physiological-visual based concept of the RGB space. With the projection it is, contrary to the prevailing opinion, possible to differentiate between colors based on human perception in the linear geometry of the RGB color space. This opens new possibilities in many fields of color image processing, especially in the domain of color image segmentation, where color similarity plays a major role.
\end{abstract}

Keywords: Color space theory, color similarity, color image segmentation, RGB color space, HSV color space.

\section{$1 \quad$ Introduction}

The RGB color space is an additive color space based on the three primary colors Red, Green and Blue. It goes back to Maxwell's work on color theory [1, 2], which laid the foundation for the RGB color model. This model is nowadays used in many applications such as digital cameras, digital screens, color scanners and digital images. In color image segmentation the RGB color space has an ambivalent reputation. On the one hand it is one of the commonly used color spaces [3], on the other hand several basic difficulties arise using this space for image segmentation [3, 4]. Even though the RGB space corresponds to the biological processing of colors in the human visual system, it does not seem to correlate with the human perceptual differentiation between colors [3, 4]. The HSV color space bases on the parameters Hue, Saturation and Value, which conform to the human differentiation between colors $[5,6]$. An advantage of this space is the separation of chromatic (Hue and Saturation) and achromatic (Value) information. It gives the possibility to treat color information independently from Value information. Anyhow due to the mathematical description in polar coordinates, the combined use of Hue and Saturation for color similarity 
measurements is difficult $[6,7]$. This fact is as well illustrated by the absence of approaches in color image segmentation using exclusively these two values for color similarity measurements, even though they are supposed to carry all chromatic information. As the HSV color space is derived from the RGB color space the question arises: Is there a possibility of separating chromatic from achromatic information directly in the RGB color space? In this paper we formulate a projection of RGB vectors within the RGB space which fulfils this separation. The projection has several advantages: Firstly, the linear geometry facilitates the mathematical description and numeric implementation of color similarity. Secondly, the decoding of chromatic and achromatic information reduces the 3-dimensional problem of similarity measurements in the RGB space to a 2-dimensional one and furthermore corresponds to the human perception of color similarity. Thirdly the additive character of the RGB color space can still be used to differentiate between color regions described by a certain constellation of primary values.

In the following section the mathematical basis for the projection is derived from the conversion rule between RGB and HSV color spaces. In the third section the projection is explained practically, while in the fourth section basic properties of the projection are shown and compared to the conventional use of Hue and Saturation in the HSV color space. A discussion of the results is given in section five, followed by the conclusion in the sixth section.

\section{Hue, Saturation and Value in the RGB Color Space}

To understand the representation of Hue, Saturation and Value in the RGB color space, their mathematical description within the geometry of the RGB color space has to be analyzed. In this section it will be shown under which conditions Hue and Saturation stay constant, while RGB values are changed. The result is the geometrical equivalent of Value within the RGB space. With this result it is possible to formulate a projection which suppresses Value information within the RGB space while preserving Hue and Saturation, which is described in section 3.

The conversion of RGB vectors to the HSV space is commonly denoted as:

$$
\begin{gathered}
R, G, B \in[0,1] ; M A X:=\max (R, G, B) ; M I N:=\min (R, G, B) \\
H: \begin{cases}0, & \text { if } R=G=B \\
60^{\circ} *\left(0+\frac{G-B}{M A X-M I N}\right), & \text { if } M A X=R \\
60^{\circ} *\left(2+\frac{B-R}{M A X-M I N}\right), & \text { if } M A X=G \\
60^{\circ} *\left(4+\frac{R-G}{M A X-M I N}\right), & \text { if } M A X=B\end{cases} \\
S:\left\{\begin{array}{c}
0, \\
\frac{M A X-M I N}{M A X}, \text { else } \\
V:=M A X
\end{array}\right.
\end{gathered}
$$


The following derivation will be done for the second case of Eq. 1 assuming that Red is the maximum of the RGB vector and Blue is the minimum. Any other case can be proven analogously.

At first the condition under which Hue stays constant when RGB values are changed has to be defined. Changing RGB values ([R1, G1, B1]' to [R2, G2, B2]') while Hue stays constant means according to Eq. 1:

$$
\frac{G_{1}-B_{1}}{R_{1}-B_{1}}=\frac{G_{2}-B_{2}}{R_{2}-B_{2}}
$$

which can be written as:

$$
\left(G_{1}-B_{1}\right) *\left(R_{2}-B_{2}\right)-\left(R_{1}-B_{1}\right) *\left(G_{2}-B_{2}\right)=\left(\begin{array}{l}
G_{1}-B_{1} \\
B_{1}-R_{1} \\
R_{1}-G_{1}
\end{array}\right) \cdot\left(\begin{array}{l}
R_{2} \\
G_{2} \\
B_{2}
\end{array}\right)=0
$$

which can also be written as:

$$
\left[\left(\begin{array}{l}
R_{1} \\
G_{1} \\
B_{1}
\end{array}\right) \times\left(\begin{array}{l}
1 \\
1 \\
1
\end{array}\right)\right] \cdot\left(\begin{array}{l}
R_{2} \\
G_{2} \\
B_{2}
\end{array}\right)=\operatorname{det}\left(\begin{array}{lll}
R_{1} & 1 & R_{2} \\
G_{1} & 1 & G_{2} \\
B_{1} & 1 & B_{2}
\end{array}\right)=0 .
$$

The term on the left means that all RGB vectors with the same Hue lie on a plane trough origin with a normal defined by the cross product between one of these RGB

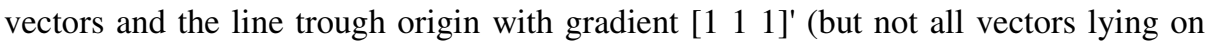
the plane have the same Hue, only those on the plane complying the postulated maximum and minimum assignments). The term is a triple product for which the identity with the determinant between the two equal signs applies. As the determinant is zero Hue stays constant while RGB values are changed if and only if two scalars $a$ and $b$ exist such as

$$
\left(\begin{array}{l}
R_{1} \\
G_{1} \\
B_{1}
\end{array}\right)+a *\left(\begin{array}{l}
1 \\
1 \\
1
\end{array}\right)+b *\left(\begin{array}{l}
R_{2} \\
G_{2} \\
B_{2}
\end{array}\right)=\left(\begin{array}{l}
0 \\
0 \\
0
\end{array}\right) ; a, b \in \mathbb{R} ; b \neq 0 .
$$

This equation holds for every possible maximum and minimum assignment.

Now the condition under which Saturation stays constant while RGB values are changed has to be defined, which means according to Eq. 2:

$$
\frac{M A X_{1}-M I N_{1}}{M A X_{1}}=\frac{M A X_{2}-M I N_{2}}{M A X_{2}} \widehat{=} \frac{M I N_{1}}{M A X_{1}}=\frac{M I N_{2}}{M A X_{2}} .
$$

That means Saturation stays constant while RGB values are changed if and only if the proportional relation between the maximum and the minimum stays constant.

To see under which condition Hue and Saturation both stay constant Eq. 7 and Eq. 8 have to be combined. As the maximum and minimum assignments cannot change (otherwise Hue would change), Saturation stays constant if

$$
\frac{B_{1}}{R_{1}}=\frac{B_{2}}{R_{2}} \hat{=} B_{1} * R_{2}=R_{1} * B_{2} .
$$


From Eq. 7 together with Eq. 9 we obtain after two intermediate steps

$$
b *\left(B_{1} * R_{2}-R_{1} * B_{2}\right)=0=a *\left(R_{1}-B_{1}\right),
$$

which is due to the maximum and minimum assignments (Red unequal Blue) only possible if $a$ is equal zero. That leads with Eq. 7 finally to the conclusion: If RGB values are changed, Hue and Saturation both stay constant if and only if a scalar $k$ exists such as

$$
\left(\begin{array}{l}
R_{1} \\
G_{1} \\
B_{1}
\end{array}\right)=k *\left(\begin{array}{l}
R_{2} \\
G_{2} \\
B_{2}
\end{array}\right) \quad ; \quad k \in \mathbb{R} .
$$

This equation holds as well for every possible maximum and minimum assignment. Eq. 11 means Hue and Saturation stay constant while RGB values are changed if the RGB vectors stay on the same line through origin. In other words: Changing Value of an RGB vector while Hue and Saturation stay constant displaces the vector along a line through origin and the original RGB vector.

\section{Projecting RGB Vectors to Separate Chromatic from Achromatic Information in the RGB Color Space}

The former conclusion is the basis for the projection of RGB vectors in the RGB space. According to Eq. 11 displacing an RGB vector along the line through origin and the original RGB vector does not change Hue or Saturation but Value. It means Hue and Saturation are defined by the gradient of the line, while Value is defined by the position of the RGB vector on the line. Suppressing Value in the RGB space can be done by neglecting the position of an RGB vector on the line through origin and itself, while conserving the information of the gradient of the line. In Fig. 1 the RGB color space is shown together with a grey shaded plane through the point $\left[\begin{array}{lll}255 & 0 & 0\end{array}\right]$ ' with normal $\left[\begin{array}{lll}1 & 1 & 1\end{array}\right]$ '. Every line through origin in the RGB space has an intersection with this plane. The intersection point of a line and the plane can be seen as a bijective mapping of the line (or the gradient of the line) on the plane: Every line has a unique intersection point on the plane and every intersection point on the plane has a unique line it represents.

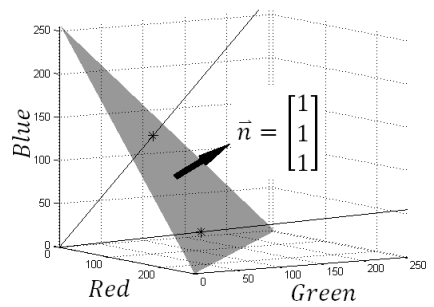

Fig. 1. Every line through origin in the RGB space intersects in a unique point a plane through point [255 00 0]' with normal [lllll 111 ' 
Projecting an RGB vector along a line through origin and itself to the plane does not affect the information of Hue and Saturation (see Eq. 11). This means a projection of all RGB vectors in the RGB space along lines through origin and each vector to the plane suppresses Value information while conserving the information of Hue and Saturation. Please note that any other plane in the RGB space, as long as its normal is [ $\left.\begin{array}{lll}1 & 1 & 1\end{array}\right]$ ' would not change the properties of the projection but resize it. The projection of an RGB vector can be mathematically described as

$$
\left(\begin{array}{l}
R \\
G \\
B
\end{array}\right)^{\prime}=\left(\begin{array}{l}
R \\
G \\
B
\end{array}\right) *\left(\begin{array}{l}
C \\
C \\
C
\end{array}\right) ; c=\frac{255}{R+G+B} .
$$

In Fig. 2 an example set of RGB vectors is projected to the mentioned plane.

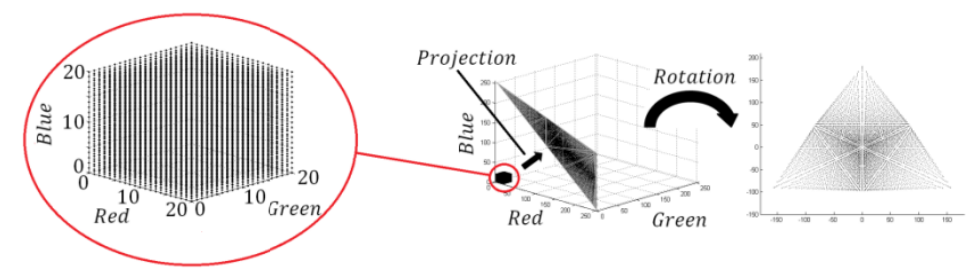

Fig. 2. An example set of RGB vectors (left) is projected to a plane through point [255 00 ]' with normal $\left[\begin{array}{lll}1 & 1 & 1\end{array}\right]$ ' (middle). The projection points are than rotated into the viewport (right).

As the projection is a 2-dimensional mapping, the projected vectors can be rotated without changing their properties (Fig. 2 on the right). The result is the 2-dimensional equivalence of Hue and Saturation in the RGB geometry. Fig. 3 on the left shows the projection of the former example vector set and additionally the projection of all RGB vectors lying on the three planes spanned by the axes of the RGB space. The additional vectors help to understand the general nature of the projection. All RGB vectors lying on the three planes spanned by the axes of the RGB space define the outer boundaries of the projection. The three corners of the projection are the intersections of the three axes of the RGB space (therefor named R, G and B). The projection of the example set is used in subsequent figures as representation of a barycentric coordinate system [8]. On the right of Fig. 3 is a real colored equivalence of the projection, which shows the general representation of colors in the projection.

In the following section the basic properties of the projection will be shown and compared to the conventional use of Hue and Saturation in the HSV space.
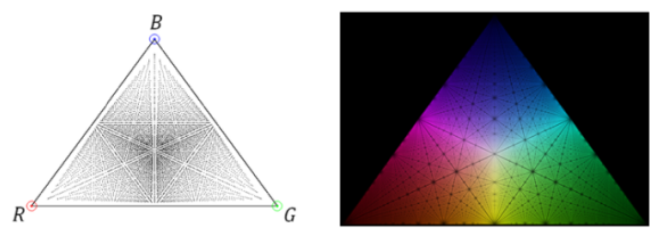

Fig. 3. The projection of an example set of RGB vectors (left) and a colored visualization of the projection (right). Please note that the image on the right contains more projection points. 


\section{$4 \quad$ Properties of the Projection}

In Fig. 4 three lines of constant Hue are shown in the projection as well as in the 2dimensional representation of Hue and Saturation in the HSV geometry (the two images on the left). Hue of an RGB vector is defined by the angle between a reference line perpendicular to the achromatic line through the Red axes and a line perpendicular to the achromatic line through the RGB vector. As the projection plane is as well perpendicular to the achromatic line, Hue in the HSV space has an exact equivalent in the projection. The general geometrical conversion between the two spaces, the transformation of the RGB cube to the HSV cylinder, is illustrated by the Magenta line of constant Saturation in both spaces (the two images on the right).
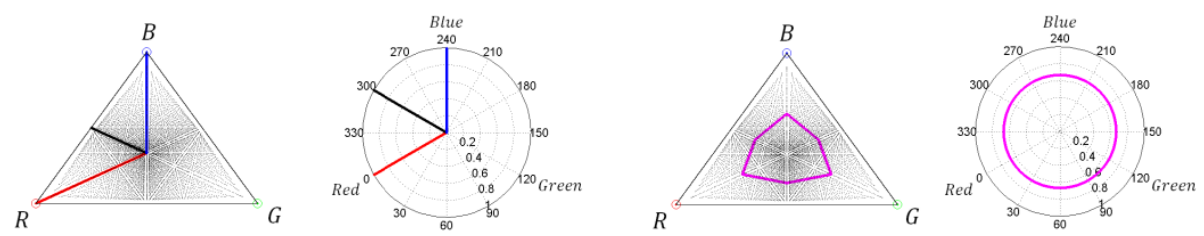

Fig. 4. Lines of constant Hue (two images on the left) and constant Saturation (two images on the right) in the projection and in the HS representation of the HSV geometry

One drawback of the HSV space is the problematic combined use of Hue and Saturation for color difference measurements as the first is an angle and the second is a radius. On the other hand Hue and Saturation are correlated, which means color difference is a function depending on both Hue and Saturation [7, 9]. In Fig. 5 different lines in the projection are shown, each one dividing the projection in two regions. As the projection can be seen as a barycentric coordinate system, each line defines a proportional relation between at least two of the primary values. On the other hand the projection represents the chromatic information of RGB vectors, which means the lines separate the projection in color regions based on the additive character of the RGB space and furthermore correspond to the HS model of human perception. In the middle of Fig. 5 are the corresponding lines in the HS geometry. It can be seen that they describe nonlinear functions that depend on both Hue and Saturation. It means the projection allows furthermore considering the correlation between Hue and Saturation with functions that are linear in the RGB color space.
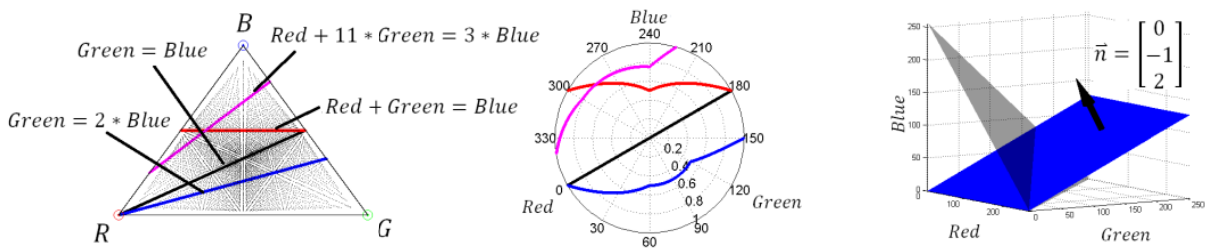

Fig. 5. Different lines in the projection (left) and their equivalent in the HSV geometry (middle). The blue plane on the right corresponds to the blue line in the projection (Green=2*Blue). 

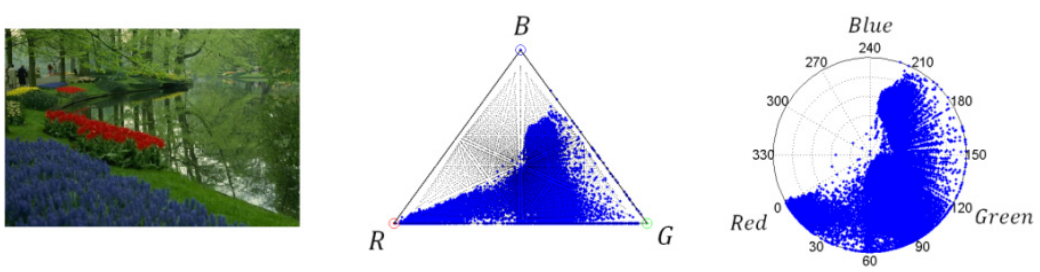

Fig. 6. An example image (left), the projection of all RGB vectors of the image (middle) and the corresponding Hue and Saturation representation in the HSV geometry (right)

One first conclusion of this concept is visualized on the right side of Fig. 5. Based on the geometrical relations explained in the previous sections it can be deviated that each continuous line in the projection is the projection of a plane through origin in the RGB space. It means each plane through origin in the RGB space divides the space based on pure chromatic information in the definition of the HSV color model. This is a result of interest for color image segmentation. In Fig. 6 an example image of the Berkeley Image Database is shown [10]. In the middle and on the right the projection of all RGB vectors and the corresponding representation in the HS geometry can be seen. Based on the explained concept four planes through origin are used to segment the color image in different regions. In Fig. 7 the segmentation results are shown together with the representation of the chosen planes in the projection. It can be seen that the image could be segmented in color regions that conform to the human perception of color homogeneity. The result visualizes the conclusions that can be obtained from the previous discussion: A separation of chromatic and achromatic information is possible in the RGB space as well as a differentiation between colors based on human perception. A further application possibility is the use of linear SupportVector Machine to obtain separation lines based on the additive character of the RGB space to classify color objects represented in the projection. Evaluating the possibilities for color image segmentation and classification is part of our current research.
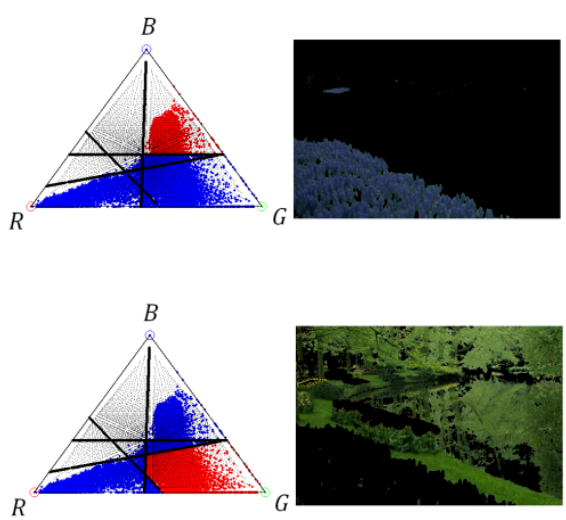
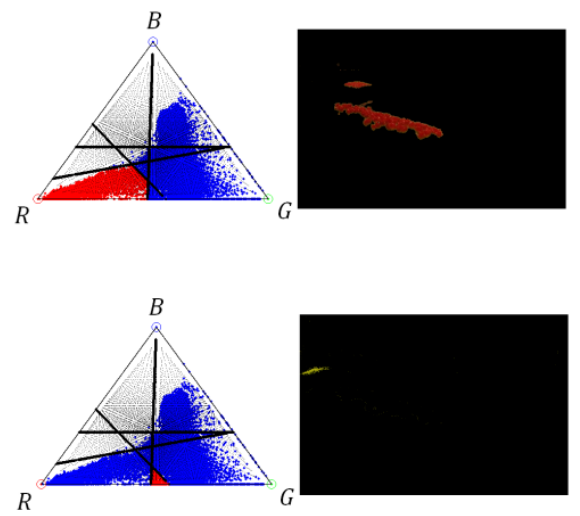

Fig. 7. Several segmentation results of the example image. On the left side of each image are in Red color the projection points which represent the segmented regions of the original image. 


\section{Discussion}

As the HS spaces are standard spaces in color image processing the geometrical relations described in section two and three are basically known. However a consistent theory which focusses on the chromatic properties of the RGB space had been missing so far. In [11] the property expressed by Eq. 11 is stated verbally without any derivation. A projection of RGB vectors to the unity plane as intermediate step of the conversion from RGB to HSI (Hue, Saturation, Intensity) equivalent to Eq. 12 is done. From this projection, known as chromaticity diagram, a two dimensional representation is obtained by only considering two components of the three dimensional projected vectors. It is the equivalence of again projecting the projection to one of the planes spanned by two of the RGB axis. This second projection is used to explain the derivation of the HSI color space, which distorts the interpretation of the global geometrical relation between the RGB and HS color spaces. In [12] the chromaticity diagram and a verbal description of the results of section two can be found as well, but no global relation to any of the HS color spaces is given. In [13] a detailed verbal derivation of section two is given but a projection or a chromaticity diagram for a separation of chromatic and achromatic information in the RGB space is not mentioned. The chromatic information of the HSI space is related to the CIE (Commission Internationale de l'Éclairage) chromaticity diagram which bases on the projected chromaticity diagram already mentioned. The tutorials section of the book website provides additional information. Here the chromaticity diagram, which is called the HSI color triangle, is shown within the RGB color space geometry. Anyhow the tutorial focusses on the explanation of the HSI color space and misses to refer back to the chromatic properties of the RGB space. It can be generally observed that in case the topic of converting from RGB to an HS color space is described, the focus lies on the properties of the HS space, without pointing back to the chromatic properties of the RGB color space. One goal of this paper is to fill this gap. The shown inconsistency in the standard literature might be one of the reasons why interpretations similar to "The HSV color space is fundamentally different from the widely known RGB color space since it separates out the Intensity (luminance) from the color information (chromaticity)." can be found in scientific papers [9]. This statement implicates that it is not possible to separate chromatic from achromatic information in the RGB color space, an interpretation that has been proven wrong in the previous sections. Furthermore interpretations like "It is more natural for human visual system to describe a color image by the HSV model than by the RGB model. Intuitively, the features extracted in the HSV color space can capture the distinct characteristics of computer graphics better." or "The first step in colour inspection was to transform the RGB information to the Hue-Lightness-Saturation (HLS) colour space. The HLS space was selected because it defines colour not only in the sense of perceptual uniformity, but more significantly, it matches to the way that the human perceives colour." or "In our approach we consider hue and saturation as discriminating color information. These attributes are strongly related to the human perception of color. In the HSV color representation, hue has the greatest discrimination power among the other coordinates." are widely spread [15-17]. Clearly Hue and Saturation are the chromatic 
parameters that are used by humans to describe or differentiate between different colors, but the common anticipation that these parameters can only be used within the HS geometry builds man-made barriers that restrict researchers from finding solutions outside the HS spaces. Statements in the standard literature such as "We can summarize that $R G B$ is ideal for color image generation [...], but its use for color image description is much more limited." support these restrictions [11]. Generally it can be stated that as soon as color information based on human perception has to be analyzed a transformation to the HS geometry with all its disadvantages is considered inevitable. It is our intention to clarify the misinterpretation that using the HS color model has to be done in an HS color space and to show that the chromatic properties of the RGB color space still carry unexploited but promising possibilities for color image processing. An example of these possibilities was given at the end of the previous section. Anyhow, as the whole method bases on the RGB geometry, the known drawbacks of this space such as its non-uniformity stay the same [6, 14]. If uniformity is a crucial parameter in a certain application, uniform color spaces such as CIE $1 * a * b$ or CIE $1 * u * v$ are so far a better choice $[6,14]$. If an application demands a consideration of Hue and Saturation independently the conventional use of the HS spaces is more efficient. Furthermore the shown theory bases on a separation of chromatic and achromatic information. A combined consideration that integrates the HS model in the RGB space for color image processing can for example be found in [18].

\section{Conclusion}

In the present paper the chromatic properties of the RGB color space based on the chromaticity definition of the HSV color space were analyzed. The equivalence of Hue and Saturation of the HSV space within the RGB geometry was formulated. From this mathematical basis a projection was deviated which separates chromatic from achromatic information in the RGB color space. The representation of chromatic information in the RGB space was compared to the conventional one of the HSV space. The contribution of the paper is the following:

- A consistent mathematical formulation and analysis of the chromatic properties of the RGB space based on the chromaticity definition of the HSV space, so far missing in the standard literature, was given.

- Based on this formulation it was shown that chromatic information can be separated from achromatic information directly in the RGB space, a possibility that is often implicitly neglected in the literature.

- It could be shown that it is contrary to the prevailing opinion possible to mathematically differentiate between color regions based on human perception directly in the RGB color space.

- A possibility of formulating linear separations of the RGB color space based on chromatic information for color image segmentation purposes was given. These linear separations describe non-linear functions in the HSV color space, depending on both Hue and Saturation. Furthermore they correspond to the human processing of visible color and correlate with the human perception of color. 


\section{References}

1. Maxwell, J.C.: Experiments on Colour as Perceived by the Eve with Remarks on ColourBlindness. Transactions of the Royal Society of Edinburgh, XXI, Part II. Edinburgh (1855)

2. Maxwell, J.C.: On the Theory of Compound Colors, and the Relations of the Colours of the Spectrum. Philosophical Transactions of the Royal Society of London 150, 57-84 (1860)

3. Cheng, H., et al.: Color Image Segmentation: Advances and Prospects. Pattern Recognition 34(12), 2259-2281 (2001)

4. Wuerger, S.M., Laurence, T.M., Krauskop, J.: Proximity Judgments in Color Space: Tests of a Euclidean Color Geometry. Vision Research 35(6), 827-835 (1995)

5. Joblove, G.H., Greenberg, D.: Color Spaces for Computer Graphics. ACM SIGGRAPH Computer Graphics 12(3) (1978)

6. Plataniotis, K.N., Venetsanopoulos, A.N.: Colour Image Processing and Applications. Springer (2000)

7. Rotaru, C., Graf, T., Zhang, J.: Color Image Segmentation in HSI Space for Automotive Applications. Journal of Real-Time Image Processing 3(4), 311-322 (2008)

8. Möbius, A.F.: Der Barycentrische Calcul (The Barycentric Calculus). Verlag von Ambrosius Barth, Leipzig (1827)

9. Sural, S., Qian, G., Pramanik, S.: Segmentation and Histogram Generation using the HSV Color Space for Image Retrieval. In: Proceedings 2002 International Conference on Image Processing, vol. 2, pp. II-589. IEEE Press, New York (2002)

10. Martin, D., Fowlkes, C., Tal, D., Malik, J.: A Database of Human Segmented Natural Images and its Application to Evaluating Segmentation Algorithms and Measuring Ecological Statistics. In: Proceedings Eighth IEEE International Conference on Computer Vision ICCV 2001, vol. 2, pp. 416-423. IEEE Press, New York (2001)

11. Jähne, B.: Digital Image Processing. Springer, Berlin (2002)

12. Wyszecki, G., Stiles, W.: Color Science. John Wiley \& Sons, New York (2000)

13. Gonzales, R., Woods, R.: Digital Image Processing. Pearson Education International, New Jersey (2008)

14. Acharya, T., Ray, A.: Image Processing. John Wiley \& Sons, New York (2005)

15. Chen, W., Shi, Y., Xuan, G.: Identifying Computer Graphics using HSV Color Model and Statistical Moments of Characteristic Functions. In: 2007 International Conference on Multimedia and Expo, pp. 1123-1126. IEEE Press (2007)

16. Nashat, S., Abdullah, M.: Multi-class Colour Inspection of Baked Foods Featuring Support Vector Machine and Wilk's lambda analysis. Journal of Food Engineering 101(4), 370-380 (2010)

17. Vitabile, S., Pollaccia, G., Pilato, G., Sorbello, F.: Road Signs Recognition using a Dynamic Pixel Aggregation Technique in the HSV Color Space. In: Proceedings 11th International Conference on Image Analysis and Processing, pp. 572-577. IEEE Press (2001)

18. Vertan, C., Zamfir, M., Zaharescu, E., Buzuloiu, V., Fernandez-Maloigne, C.: Nonlinear Color Image Filtering by Color to Planar Shape Mapping. In: 2003 International Conference on Image Processing, vol. 1, pp. 885-888. IEEE Press (2003) 